\title{
Tumour angiogenesis is reduced in the Tc1 mouse model of Down Syndrome
}

\author{
Louise E. Reynolds ${ }^{1}$, Alan R. Watson ${ }^{1,=}$, Marianne Baker ${ }^{1,=}$, Tania A. Jones ${ }^{2 a}$, Gabriela \\ D’Amico $^{1}$, Stephen D. Robinson ${ }^{1}$, Carine Joffre ${ }^{1 a}$, Sarah Garrido-Urbani ${ }^{3}$, Juan Carlos \\ Rodriguez-Manzaneque ${ }^{4}$, Estefanía Martino-Echarri ${ }^{4}$, Michel Aurrand-Lions ${ }^{5}$, Denise \\ Sheer $^{2 a}$, Franca Dagna-Bricarelli ${ }^{6}$, Dean Nizetic ${ }^{2 b}$, Christopher J. McCabe ${ }^{7 a}$, Andrew S. \\ Turnell $^{7 b}$, Stephanie Kermorgant ${ }^{1 \mathrm{a}}$, Beat A. Imhof ${ }^{3}$, Ralf Adams ${ }^{8}$, Elizabeth M.C. Fisher ${ }^{9}$, \\ Victor L. J. Tybulewicz ${ }^{10}$, lan R. Hart ${ }^{1 a}$, and Kairbaan M. Hodivala-Dilke ${ }^{1}$
}

${ }^{1}$ Centre for Tumour Biology, Barts Cancer Institute - a CR-UK Centre of Excellence, Queen Mary University of London, John Vane Science Centre, Charterhouse Square, London, EC1M 6BQ ${ }^{1 a}$ Centre for Tumour Biology, Institute of Cancer, Barts \& The London School of Medicine \& Dentistry, Queen Mary University of London, John Vane Science Centre, Charterhouse Square, London EC1M 6BQ, UK ${ }^{2 a}$ Neuroscience Centre, Barts \& The London School of Medicine \& Dentistry, Queen Mary University of London, Institute of Cell and Molecular Sciences, 4, Newark Street, London E1 2AD, UK 2b Paediatrics Centre, Barts \& The London School of Medicine \& Dentistry, Queen Mary University of London, Institute of Cell and Molecular Sciences, 4, Newark Street, London E1 2AD, UK ${ }^{3}$ Department of Pathology \& Immunology, University of Geneva, CMU rue Michel Servet1 CH-1211, Geneva, Switzerland ${ }^{4}$ GENYO Avda. Del Conocimiento, s/n Armilla 18100, Granada, Spain ${ }^{5}$ INSERM 27, Bd Lei Roure, 13009 Marseille, France ${ }^{6}$ Human Genetics Institute, Galliere Hospital, Via Volta 1016128 Genoa, Italy 7aSchool of Clinical and Experimental Medicine, University of Birmingham, Birmingham B15 2TT, UK ${ }^{7 b}$ School of Cancer Sciences, University of Birmingham, Birmingham B15 2TT, UK ${ }^{8}$ Max Planck Institute for Molecular Biomedicine, Röntgenstr. 20, D-48149 Münster, Germany ${ }^{9}$ Dept. of Neurodegenerative Disease, UCL Institute of Neurology, Queen Square, London WC1N 3BG, UK ${ }^{10}$ Division of Immune Cell Biology, MRC National Institute for Medical Research, The Ridgeway, Mill Hill, London NW7 1AA, UK

\section{Abstract}

Down Syndrome (DS) is a genetic disorder caused by full or partial trisomy of chromosome 21 . It occurs in approximately $1 / 750$ live births and presents with many clinical phenotypes including a reduced incidence of solid tumours ${ }^{1,2}$. Recent work using the Ts65Dn model of DS, that has

\footnotetext{
Correspondence and requests for material should be addressed to 1.reynolds@qmul.ac.uk. .

$=$ These authors contributed equally to the paper

Supplementary Information is linked to the online version of the paper at www.nature.com/nature

Author Contributions L.E.R. and K.M.H-D. designed the experiments. L.E.R. performed the experiments. A.R.W. performed the bone marrow transplant experiments and stained for Y chromosome and carried out RT-PCR. G.D'A. performed the aortic ring assay. S.D.R. performed the phospho-VEGFR2 Western blot analysis. T.A.J. and D.S. performed the tumour cell karyotyping. M.B. assisted with the immunostaining, tumour and sponge harvesting and flow cytometric analysis. C.J. and S.K. carried out flow cytometry and immunofluorescence of cells. B.A.I., R.A. and S.G.-U. supplied the JAM-B antibodies for Western blot analysis and JAM-B wild-type and heterozygous mice for in vivo and ex vivo studies and JAM-B biochemistry in JAM-B heterozygotes. JC.R-M. and E.M.-E. provided the ADAMTS1-heterozygous aortae and JAM-B PCR analysis. C.J.M. and A.T. provided the PTTG1IP antibody for Western blot analysis. F.D-B. and D.N. provided the human DS and normal control cells. V.J.T. and E.M.C.F. designed and developed the Tc1 mouse. L.E.R. and I.R.H. wrote the paper with substantial input from the authors.

Author Information Reprints and permissions information is available at npg.nature.com/reprintsandpermissions.

The authors declare no competing financial interests.
} 
orthologs of approximately $50 \%$ of the genes on human chromosome 21 (Hsa21), has suggested that three copies of the ETS $2^{3}$ or Down Syndrome candidate region 1 (DSCRI) genes ${ }^{4}$ (a previously known suppressor of angiogenesis ${ }^{5,6}$ ) is sufficient to inhibit tumour growth. We have used the Tc1 transchromosomic mouse model of $\mathrm{DS}^{9}$ to dissect the contribution of extra copies of genes on Hsa21 to tumour angiogenesis. This mouse expresses approximately $81 \%$ of Hsa21 genes but not the human DSCR1 region (Supplementary Fig. 1). We transplanted B16F0 and Lewis Lung Carcinoma (LLC) tumour cells into Tc1 mice and showed that growth of these tumours was reduced substantially when compared to wild-type littermate controls. Furthermore, tumour angiogenesis was repressed significantly in Tc1 mice. In particular, in vitro and in vivo angiogenic responses to vascular endothelial growth factor (VEGF) were inhibited. Examination of the genes on the segment of $\mathrm{Hsa} 21 \mathrm{in} \mathrm{Tc1}$ mice identified putative anti-angiogenic genes $\left(A D A M T S 1^{7,8}\right.$ and $E R G^{9}$ ) and novel endothelial cell-specific genes ${ }^{10}$, never shown before to be involved in angiogenesis $\left(J A M-B^{11}\right.$ and PTTG1IP) that, when overexpressed, are responsible for the inhibition of angiogenic responses to VEGF. Three copies of these genes within the stromal compartment reduced tumour angiogenesis providing an explanation for the reduced tumour growth in DS. Furthermore, we anticipate that, in addition to the candidate genes that we show to be involved in the repression of angiogenesis, the Tc1 mouse model of DS will likely allow for the identification of other endothelial-specific anti-angiogenic targets relevant to a broad spectrum of cancer patients.

Down Syndrome, or trisomy 21, is responsible for developmental abnormalities, mental retardation, cardiovascular defects and dementia ${ }^{1}$. As well as these phenotypes, which have been well documented in DS, three independent studies have drawn attention to the fact that malignant solid tumours are under-represented when compared to age- and sex-matched individuals from the general population $1,2,12$. This phenotype is likely due to "gene dosage" effects i.e. an extra copy of Hsa21 leads to higher levels of gene product that protect DS individuals from solid tumour growth.

Orthology to Hsa21 is found on three mouse chromosomes: Mmu16, Mmu17 and Mmu10, with 29 genes found on Hsa21 not being represented in the mouse (Supplementary Fig. 1). Early transgenic mouse models, including the Ts65Dn model which contains orthologs of up to only $50 \%$ of the genes located on Hsa21, have been used to study the reduced solid tumour malignancies ${ }^{3}$. However, the contribution of trisomy 21 specifically to angiogenesis, a process essential for solid tumour growth was not possible to examine in this system since all cells, including both the tumour cells and stromal cells, were potentially trisomic. We used the Tc1 mouse model of DS that has $81 \%$ of Hsa21 genes, but lacks duplication of 29 genes including DSCR1, to test specifically the effect of this additional segment of chromosome 21 on tumour neovascularisation (Supplementary Fig. 1).

To demonstrate whether Tc1 mice were protected from solid tumour growth, we examined the growth of B16F0 and LLC tumour xenografts. Tumour growth of both B16F0 and LLC was significantly repressed in Tc1 mice when compared to wild-type controls (Fig. 1a). Additionally, LLC tumour growth kinetics were significantly slower in Tc1 mice especially at larger tumour sizes which require neovascularisation rather than vessel co-option ${ }^{13}$ (Supplementary Fig. 2). To confirm this phenotype was not the result of a disproportionate increase of Mmu 10,16 and 17 relative to the ploidy of the tumour cells, we examined the chromosomes in the B16F0 and LLC cell lines. Mmu 10, 16 and 17 were found to be correct relative to the ploidy (Supplementary Fig. 3). These observations confirm that three copies of chromosome 21 genes suppress the growth of tumours through reduced tumour neoangiogenesis.

To address specifically the contribution of tumour angiogenesis to this phenotype, the tumour vasculature was examined. The additional Hsa21 fragment in Tc1 mice induced a 
significant and substantial decrease in tumour blood vessel density in both B16F0 and LLC tumours when compared to tumours grown in wild-type littermate controls (Fig. 1b). Dextran-FITC perfused blood vessel numbers were decreased also in Tc1 mouse tumours (Fig. 1c). In contrast, examination of blood vessel density in unchallenged skin and other organs from wild-type control and Tc1 mice showed no difference between the genotypes (Fig. 1d and Supplementary Fig. 4), suggesting that pathological angiogenesis, but not normal blood vessel density, is negatively affected by the Hsa21 fragment. In addition, the growth of Tc1 endothelial cells was not affected by the additional Hsa21 fragment ${ }^{14}$ (Supplementary Fig. 5). Furthermore, the differences in tumour vascularity were not due to differences in immune-cell infiltration between the genotypes (Supplementary Fig. 6) and the contribution of Tc1-bone marrow derived cells to tumour growth was not apparent (Supplementary Fig. 7). Taken together, our data from Tc1 mice suggest that it is the presence of an additional copy of a fragment of Hsa21, within blood vessels, that inhibits solid tumour growth and, importantly, represses tumour angiogenesis.

To assess whether the decreased tumour angiogenesis observed in the Tc1 mice was due to reduced sensitivity to VEGF, we performed in vivo growth factor-induced angiogenesis assays. VEGF-mediated neovascularisation was reduced significantly in the Tc1 mice when compared with littermate controls (Fig. 2a). PBS-treatment resulted in similar baseline angiogenic responses in both genotypes (data not shown). In addition to the lack of in vivo angiogenesis, Tc1 aortic rings were also unresponsive to VEGF-stimulation when compared with VEGF-treated wild-type controls. Baseline responses to PBS were not affected in the $\mathrm{Tc} 1$ aortic rings indicating further that an additional copy of the fragment of Hsa21 specifically suppresses VEGF-induced neovascularisation (Fig. 2b).

Vascular endothelial growth factor receptor 2 (VEGFR2) is a major pro-angiogenic growth factor receptor ${ }^{15}$. VEGF, via VEGFR2, induces ERK1/2 (p42/p44) phosphorylation and mediates endothelial cell activation during angiogenesis and inhibition of VEGFR2 or the ERK1/2 pathway reduces VEGF-mediated angiogenic responses ${ }^{16}$. ERK1/2 phosphorylation was reduced specifically in response to VEGF, but not basic fibroblast growth factor (bFGF), in Tc1 endothelial cells when compared with wild-type controls and in VEGFstimulated primary cells isolated from individuals with DS (Fig. 2c, d and Supplementary Fig. 8). This specific response to VEGF focused our attention on VEGFR2.

Although other molecules, such as DYRK1A, have been reported to be upstream of ERK signalling ${ }^{17}$, and may contribute to the decreased ERK-phosphorylation in response to VEGF, we show that surface levels, but not total levels, of VEGFR2 are substantially increased in Tc1 endothelial cells (Supplementary Fig. 9a, b). Interestingly, after VEGF stimulation the surface levels of VEGFR2 remain consistently higher on Tc1 endothelial cells than on control cells (Supplementary Fig. 9c). This discrepancy between total VEGFR2 and surface VEGFR2 levels identifies that Tc1 endothelial cells have lower cytoplasmic levels of VEGFR2. Indeed, immunofluorescence examination of endothelial cells in culture show that stimulation of wild-type cells with VEGF induced an apparent internalisation of phosphorylated VEGFR2 that was not present in Tc1 endothelial cells (Supplementary Fig. 9c). The phosphorylated VEGFR2 in Tc1 endothelial cells appeared to be restricted at the cell surface after VEGF-stimulation. Although beyond the scope of the study, it is tempting to speculate that defects in VEGFR2 subcellular localisation are relevant to the repressed angiogenesis in Tc1 mice and provide a novel aspect to the regulation of angiogenesis in $\mathrm{DS}^{18,19}$.

We identified several putative anti-tumourigenic, anti-angiogenic and endothelial cellspecific genes expressed on Hsa21 in the Tc1 mice likely to be responsible for the decreased angiogenic responses. These included ETS2, a transcription factor whose overexpression 
reduces tumour growth in the Ts65Dn mouse model of DS and other models ${ }^{3,20}$ but not yet linked with angiogenesis; $A D A M T S 1$, a protease known to inhibit angiogenesis ${ }^{7,8,21}$; three endothelial cell-specific Hsa21 genes: $E R G$, a transcription factor implicated in endothelial tube formation and angiogenesis ${ }^{9}, J A M-B$ a cell-cell adhesion molecule not yet implicated in angiogenesis or tumourigenesis and PTTG1IP, which is overexpressed in cancers but also not yet linked to angiogenesis. All mouse transcripts were expressed in Tc1 and wild-type endothelial cells and human transcripts only in the Tc1 endothelial cells (Supplementary Fig. $10 \mathrm{a}, 10 \mathrm{~b})$. To test whether three copies of any of these candidate genes negatively affected angiogenesis, we first observed the effects of depleting individual human transcripts by RNAi on VEGF-mediated microvessel sprouting in aortic ring assays (Fig. 3). Knockdown of the human transcripts and protein in Tc1 endothelial cells was confirmed by RTPCR and Western blot analysis and no detectable effects on mouse orthologs were observed (Fig. 3a and Supplementary Fig. 11). Wild-type aortic rings stimulated with VEGF showed a significant increase in microvessel sprouting that was not affected by treatment with scrambled (Scr) or the human-specific siRNAs against ETS2, ERG, ADAMTS1, JAM-B or $P T T G 1 I P$ (Fig. 3b). This was expected since these aortic rings lacked any human genes and acted as a control. In contrast, Tc1 aortic rings did not show enhanced microvessel sprouting in response to VEGF-stimulation with or without Scr-siRNA transfection (Fig. 3c). However, using human-specific siRNAs to deplete one out of three copies of $E R G$, ADAMTS1, JAM-B or PTTG1IP transcripts (effectively recreating wild-type copy numbers for each gene) was sufficient to restore VEGF-mediated microvessel sprouting to VEGFtreated wild-type levels. Depletion of one out of three copies of ETS2 did not induce a significant increase in microvessel sprouting in response to VEGF (Fig. 3c) suggesting that vascular ETS2 is not involved in this response. In contrast, data from Sussan et al. ${ }^{3}$ suggest that ETS2 is involved in the growth of spontaneous intestinal tumours in $\mathrm{APC}^{\mathrm{min}}$ mice. Taken together these data suggest that the effect of ETS 2 is in the non-stromal tumour cell compartment. Indeed, ETS 2 has been reported to be responsible for different biological responses in different cell types $22,23,24$. Our data provide an example of how the xenograft model used in the Tc1 mice enables us to dissect the role of genes in the tumour and stromal compartment.

To further test the gene-dosage effect of the remaining candidate genes we used mousespecific siRNAs to deplete two out of three transcript copies in Tc1 aortic rings. Using RTPCR we showed that mouse-specific siRNAs for the candidate genes Erg, Adamts1, Jam$b$ and Pttg1ip effectively depleted mouse transcripts in Tc1 endothelial cells (Fig. 4a). As in Figure 3c, VEGF-treatment of Tc1 aortic rings that were either untransfected or transfected with Scr-siRNA did not induce an increase in microvessel sprouting over untreated control aortic rings. In contrast, targeting the mouse Adamts1, Jam-b or Pttg1ip transcripts by siRNA promoted VEGF-mediated microvessel sprouting over and above Scr-siRNA treated controls. Depleting two out of three copies of these transcripts (effectively reducing the copy number of each gene from three to one) is sufficient to promote VEGF-mediated microvessel sprouting (Fig. 4b). Together, our data show that for Adamts1, Jam- $b$ and Pttglip one or two copies of the transcript is sufficient to restore normal levels of VEGFmediated vessel sprouting, suggesting that a gene dosage effect is mediating the repressed angiogenic phenotype in the Tc1 mice. In contrast depleting two out of three copies of the Erg gene did not affect VEGF-mediated vessel sprouting suggesting that two copies of this transcript are required for normal angiogenic responses.

In addition, an antibody against mouse JAM-B was injected into B16F0 tumour-burdened, Tc1 mice. Intraperitoneal administration of this antibody increased significantly tumour size and blood vessel density in Tc1 mice when compared with control-IgG injected Tc1 controls (Fig. 4c). To provide direct evidence of a gene-dosage effect of JAM-B on angiogenesis we compared angiogenesis in wild-type mice ( 2 copies of JAM-B) with JAM-B heterozygous 
mice (1 copy of JAM-B). JAM-B-heterozygous mouse tissue showed approximately a half the levels of JAM-B (Supplementary Fig. 12). B16F0 tumour size and blood vessel density was increased significantly in JAM-B-heterozygous mice when compared with wild-type mice (Fig. 4d, e). Furthermore, aortic rings from JAM-B-heterozygous mice showed a significant increase in VEGF-mediated aortic vessel sprouting (Fig. 4f). Finally, we compared VEGF-induced angiogenesis ex vivo using aortae isolated from wild-type and ADAMTS1-heterozygous mice. Once again, our data showed a gene-dosage effect of ADAMTS1 on angiogenesis and corroborate previous findings where genetic ablation of ADAMTS1 enhances angiogenesis ${ }^{25}$ (Supplementary Fig. 13). Together, these results confirm that one extra copy of the candidate genes on Hsa21 can provide an anti-angiogenic effect.

Our work establishes a novel finding that three copies of individual genes in the stromal compartment can confer a repressed tumour angiogenic phenotype in the Tc1 mouse model of DS, supporting the notion of a 'gene-dosage' effect, for some genes, protecting DS individuals from solid tumour growth ${ }^{1,2}$. In particular, VEGF-mediated angiogenic processes are inhibited by the addition of a fragment of Hsa21 in the Tc1 mice. Our data demonstrate that individual depletion either of JAM-B, ADAMTS1, ERG or PTTG1IP, but not $E T S 2$, is sufficient to restore normal angiogenic responses to VEGF. This implies that three copies of $J A M-B, A D A M T S 1, E R G$ and PTTG1IP genes, and probably others that we have not tested, need to be present for the overall inhibitory effect. The transchromosomic system used in our study has a unique advantage in allowing species-specific silencing of the third gene copy, leading to a rapid genetic dissection and pinpointing of the causative trisomic genes. Although beyond the scope of our present study, our results imply that $J A M$ $B, A D A M T S 1, E R G$ and PTTG1IP, but not ETS2, are upstream of a common signalling pathway in the control of angiogenic responses.

Although we a see a similar phenotype in the Tc1 mice as Baek et a ${ }^{4}$, the mechanisms of this regulation are likely to be different since the DSCR1 region is triplicated in the TS65Dn mice, but expressed at normal levels in the Tc1 mice. Because of this, changes in DSCR1 mediated signalling via calcineurin are unlikely to be affected in the Tc1 mice. Indeed, although the overexpression of $D S C R 1$ has been shown to inhibit tumour angiogenesis, overexpression of the same gene is also thought to contribute to several pathological defects ${ }^{26}$ suggesting that manipulation of DSCR1 downstream signalling may have limited anti-angiogenic therapeutic potential. In contrast, our studies not only confirmed the function of known anti-angiogenic genes on Hsa21 but, moreover, identified and tested novel endothelial-specific genes that are anti-angiogenic when overexpressed. Our data highlights the importance of $A D A M T S 1, J A M-B, P T T G 1 I P$ and other, potentially novel, genes in controlling tumour angiogenesis. Furthermore, we have identified $J A M-B$ and PTTG1IP as new anti-angiogenic mediators.

Our study reveals that repression of angiogenesis by addition of a fragment of Hsa21 in DS models can direct the discovery of novel anti-angiogenic targets, which have the potential of being exploited pharmacologically for the development of effective gene therapy for treating cancer.

\section{Methods}

\section{Mice}

$\mathrm{Tc}(\mathrm{Hsa} 21) 1 \mathrm{TybEmcf}$ (Tc1) mice were generated by crossing Tc1 females with (129S8 $\times$ B6)F1 males ${ }^{9}$. Experiments were carried out using male JAM-B heterozygous, Tc1 and wild-type littermate mice aged 2-4 months. 


\section{In vivo tumour assays}

$1 \times 10^{6} \mathrm{~B} 16 \mathrm{~F} 0$ melanoma cells or $0.5 \times 10^{6}$ Lewis Lung Carcinoma (LLC) cells were injected subcutaneously into the scruff of test and control mice and were measured and processed for histological analysis after 12 or 10 days of growth respectively as described previously ${ }^{45}$. For the LLC tumour kinetic experiment, $0.5 \times 10^{6}$ Lewis Lung Carcinoma (LLC) cells were injected subcutaneously into the flank of Tc1 and wild-type littermate control mice and tumour volume, $v$, was calculated by palpation (using the formula $v=0.5 \times\left(I \times W^{2}\right)$ where $I$ $=$ tumour length and $w=$ tumour width) every 2 days up to day 16 .

The anti-JAM-B antibody (clone JB4.2) was developed in Beat A. Imhof's laboratory (University of Geneva, Switzerland) and will be described elsewhere. For JAM-B blocking experiments, $100 \mu \mathrm{g}$ of JB4.2 was injected intraperitoneally every 3 days into tumourburdened Tc1 and wild-type mice. On day 12 tumours were measured and processed for histological analysis.

\section{Subcutaneous angiogenesis assay}

Assays were performed as described previously ${ }^{46}$. Sponges were implanted into the flanks of age- and sex-matched Tc1 and wild-type littermate control mice and the following day the sponges were injected directly with either VEGF (10 ng final concentration) or PBS as a control, every 3 days up to 14 days. On day 14 the sponges were harvested and fixed for immunostaining for blood vessel examination.

\section{Western blot analysis}

Wild-type and Tc1 primary endothelial cells were plated into 6 or $10 \mathrm{~cm}$ dishes and grown in MLEC-medium to $70 \%$ confluence. The medium was removed and the dishes were washed twice with PBS followed by lysis with RIPA or $1 \times$ modified sample buffer ( $3 \%$ SDS, $60 \mathrm{mM}$ sucrose, $65 \mathrm{mM}$ Tris-HCl, pH6.8). For phosphorylation studies, the cells were incubated for $4 \mathrm{~h}$ in serum free Optimem after which half the dishes were treated with $30 \mathrm{ng} /$ $\mathrm{ml}$ VEGF or $30 \mathrm{ng} / \mathrm{ml} \mathrm{bFGF} \mathrm{for} 5 \mathrm{~min}$ at $37^{\circ} \mathrm{C}$. The remainder were left untreated. The cell lysates were sonicated briefly, centrifuged and the supernatant collected for Western blot analysis. 30-100 $\mu \mathrm{g}$ protein for each protein sample was loaded onto 8-12\% gels and Western blot analysis was performed. Membranes were incubated with primary antibody at $4^{\circ} \mathrm{C}$ (VEGFR2/phospho-VEGFR2, ERK1/2 and phospho-ERK1/2 all from Cell Signaling Technology, UK). Anti-ADAMTS1 antibody was a kind gift from Luisa Iruela-Arispe, UCLA. Anti-PTTG1IP antibody was raised in rabbits against the human PTTG1IP protein. Anti-JAM-B antibody was kindly provided by Beat A. Imhof, University of Geneva). This was followed by $1 \mathrm{~h}$ incubation with the relevant secondary-HRP conjugated antibody.

\section{Aortic ring assay}

This assay was performed as described previously ${ }^{45}$. Briefly, aortae were isolated from Tc1 and wild-type littermate controls, sliced into rings and serum-starved overnight in Optimem. Aortic rings were embedded in a collagen matrix and cultured in the presence of VEGF (30 $\mathrm{ng} / \mathrm{ml}$ ), bFGF (30ng/ml) or PBS as a negative control or $10 \%$ foetal calf serum as a positive control. For RNAi studies, aortic rings were transfected with various siRNAs (Dharmacon) prior to embedding in collagen. Angiogenic sprouts were counted up to 7 days after embedding. To confirm gene knockdown whole aortic rings were transfected with RNAi for $48 \mathrm{~h}$. RNA was extracted from the aortae using the Qiagen RNeasy mini kit, reverse transcribed and the resulting cDNA was used for RT-PCR analysis. 


\section{Blood vessel quantitation}

Frozen tumour sections or paraffin-fixed sponge sections were immunostained with rat $a$ endomucin (Santa Cruz, UK) primary antibody to identify blood vessels. The number of blood vessels was counted in a double-blind manner across entire midline sections of sizematched tumours or sponges ${ }^{45}$. Blood vessel density was calculated as the number of blood vessels per unit area of section.

\section{Statistical analysis}

Statistical significance was calculated using the Students t-test. $P<0.05$ was considered statistically significant.

\section{Animal regulations}

All animals were used in accord with United Kingdom Home Office regulations.

\section{Supplementary Material}

Refer to Web version on PubMed Central for supplementary material.

\section{Acknowledgments}

The authors thank Garry Saunders, Colin Wren, Colin Pegrum and Amy Slender for their help with animal husbandry. Frances Wiseman and Tommy Broughton provided the information regarding gene deletions in the Tc1 mice. The authors also thank Luisa Iruela-Arispe (UCLA) for advice on ADAMTS1 mice.

\section{References}

1. Yang Q, Rasmussen SA, Friedman JM. Mortality associated with Down's syndrome in the USA from 1983 to 1997: a population-based study. Lancet. 2002; 359:1019-1025. [PubMed: 11937181]

2. Hasle H. Pattern of malignant disorders in individuals with Down's syndrome. Lancet Oncol. 2001; 2:429-436. [PubMed: 11905737]

3. Sussan TE, Yang A, Li F, Ostrowski MC, Reeves RH. Trisomy represses Apc(Min)-mediated tumours in mouse models of Down's syndrome. Nature. 2008; 451:73-75. [PubMed: 18172498]

4. Baek KH, et al. Down's syndrome suppression of tumour growth and the role of the calcineurin inhibitor DSCR1. Nature. 2009; 459:1126-1130. [PubMed: 19458618]

5. Iizuka M, Abe M, Shiiba K, Sasaki I, Sato Y. Down syndrome candidate region 1, a downstream target of VEGF, participates in endothelial cell migration and angiogenesis. J. Vasc. Res. 2004; 41:334-344. [PubMed: 15263820]

6. Minami T, et al. Vascular endothelial growth factor- and thrombin-induced termination factor, Down syndrome critical region-1, attenuates endothelial cell proliferation and angiogenesis. J. Biol. Chem. 2004; 279:50537-50554. [PubMed: 15448146]

7. Luque A, Carpizo DR, Iruela-Arispe ML. ADAMTS1/METH1 inhibits endothelial cell proliferation by direct binding and sequestration of VEGF165. J. Biol. Chem. 2003; 278:23656-23665. [PubMed: 12716911]

8. Lee NV, et al. ADAMTS1 mediates the release of antiangiogenic polypeptides from TSP1 and 2. EMBO J. 2006; 25:5270-5283. [PubMed: 17082774]

9. Birdsey GM, et al. Transcription factor Erg regulates angiogenesis and endothelial apoptosis through VE-cadherin. Blood. 2008; 111:3498-3506. [PubMed: 18195090]

10. Herbert JM, Stekel D, Sanderson S, Heath VL, Bicknell R. A novel method of differential gene expression analysis using multiple cDNA libraries applied to the identification of tumour endothelial genes. BMC Genomics. 2008; 9:153. [PubMed: 18394197]

11. Aurrand-Lions M, Duncan L, Ballestrem C, Imhof BA. JAM-2, a novel immunoglobulin superfamily molecule, expressed by endothelial and lymphatic cells. J. Biol. Chem. 2001; 276:2733-2741. [PubMed: 11053409] 
12. Satge D, et al. A tumor profile in Down syndrome. Am. J. Med. Genet. 1998; 78:207-216. [PubMed: 9677053]

13. Holash J, et al. Vessel cooption, regression, and growth in tumors mediated by angiopoietins and VEGF. Science. 1999; 284:1994-1998. [PubMed: 10373119]

14. Williams BR, et al. Aneuploidy affects proliferation and spontaneous immortalization in mammalian cells. Science. 2008; 322:703-709. [PubMed: 18974345]

15. Shalaby F, et al. Failure of blood-island formation and vasculogenesis in Flk-1-deficient mice. Nature. 1995; 376:62-66. [PubMed: 7596435]

16. Reynolds AR, et al. Elevated Flk1 (vascular endothelial growth factor receptor 2) signaling mediates enhanced angiogenesis in beta3-integrin-deficient mice. Cancer Res. 2004; 64:86438650. [PubMed: 15574772]

17. Kelly PA, Rahmani Z. DYRK1A enhances the mitogen-activated protein kinase cascade in PC12 cells by forming a complex with Ras, B-Raf, and MEK1. Mol. Biol. Cell. 2005; 16:3562-3573. [PubMed: 15917294]

18. Gampel A, et al. VEGF regulates the mobilization of VEGFR2/KDR from an intracellular endothelial storage compartment. Blood. 2006; 108:2624-2631. [PubMed: 16638931]

19. Lampugnani MG, Orsenigo F, Gagliani MC, Tacchetti C, Dejana E. Vascular endothelial cadherin controls VEGFR-2 internalization and signaling from intracellular compartments. J. Cell Biol. 2006; 174:593-604. [PubMed: 16893970]

20. Wolvetang EJ, et al. ETS2 overexpression in transgenic models and in Down syndrome predisposes to apoptosis via the p53 pathway. Hum. Mol. Genet. 2003; 12:247-255. [PubMed: 12554679]

21. Liu YJ, Xu Y, Yu Q. Full-length ADAMTS-1 and the ADAMTS-1 fragments display pro- and antimetastatic activity, respectively. Oncogene. 2006; 25:2452-2467. [PubMed: 16314835]

22. Carbone GM, et al. Triplex DNA-mediated downregulation of Ets 2 expression results in growth inhibition and apoptosis in human prostate cancer cells. Nucleic Acids Res. 2004; 32:4358-4367. [PubMed: 15314206]

23. Yamamoto H, et al. Defective trophoblast function in mice with a targeted mutation of Ets2. Genes Dev. 1998; 12:1315-1326. [PubMed: 9573048]

24. Park JS, et al. A role for both Ets and C/EBP transcription factors and mRNA stabilization in the MAPK-dependent increase in $\mathrm{p} 21$ (Cip-1/WAF1/mda6) protein levels in primary hepatocytes. Mol. Biol. Cell. 2000; 11:2915-2932. [PubMed: 10982390]

25. Shozu M, et al. ADAMTS-1 is involved in normal follicular development, ovulatory process and organization of the medullary vascular network in the ovary. J. Mol. Endocrinol. 2005; 35:343355. [PubMed: 16216914]

26. Harris CD, Ermak G, Davies KJ. Multiple roles of the DSCR1 (Adapt78 or RCAN1) gene and its protein product calcipressin 1 (or RCAN1) in disease. Cell Mol. Life Sci. 2005; 62:2477-2486. [PubMed: 16231093]

27. O’Doherty A, et al. An aneuploid mouse strain carrying human chromosome 21 with Down syndrome phenotypes. Science. 2005; 309:2033-2037. [PubMed: 16179473]

28. Reynolds AR, et al. Stimulation of tumor growth and angiogenesis by low concentrations of RGDmimetic integrin inhibitors. Nat. Med. 2009; 15:392-400. [PubMed: 19305413]

29. Reynolds LE, et al. Enhanced pathological angiogenesis in mice lacking beta3 integrin or beta3 and beta5 integrins. Nat. Med. 2002; 8:27-34. [PubMed: 11786903]

30. Mahadevan V, Hart IR, Lewis GP. Factors influencing blood supply in wound granuloma quantitated by a new in vivo technique. Cancer Res. 1989; 49:415-419. [PubMed: 2463076] 

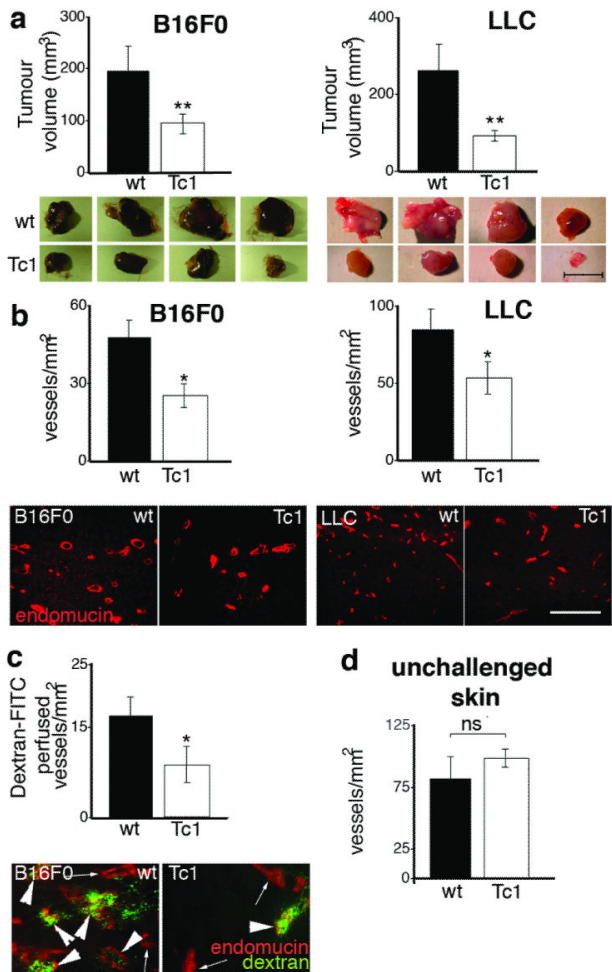

d unchallenged

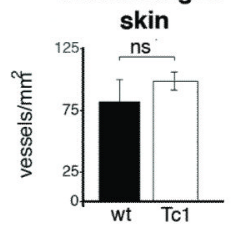

Figure 1. Tumour angiogenesis is restricted in Tc1 mice

a, B16F0 and LLC tumour size was reduced significantly in the Tc1 mice. Representative tumour images are given. $n=20$ per group. b, Blood vessel density was reduced significantly in tumours from Tc1 mice. Representative staining for endomucin in tumour sections is given. $n=5-10$ per group. $\mathbf{c}$, Perfusion of tumour blood vessels in Tc1 mice was reduced when compared with wild-type (wt) controls. Graph shows mean number of dextran-FITC perfused blood vessels. Arrow heads, perfused vessels; arrows, non-perfused vessels. d, Blood vessel density was similar in unchallenged skin of wild-type (wt) and Tc1 mice. $n=5$ per group. $* * \mathrm{P}<0.05, * \mathrm{P}<0.01$, ns=not statistically significant. Scale bars: $10 \mathrm{~mm}(\mathrm{a}) ; 100$ $\mu \mathrm{m}$ (b). All values are means \pm SEM. 

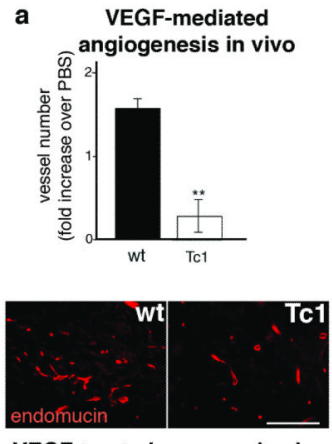

VEGF-treated sponges in vivo

c
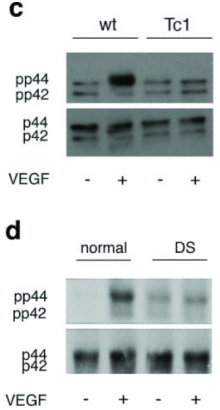
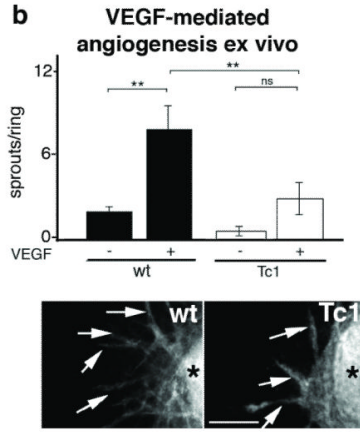

VEGF-treated aortic rings ex vivo
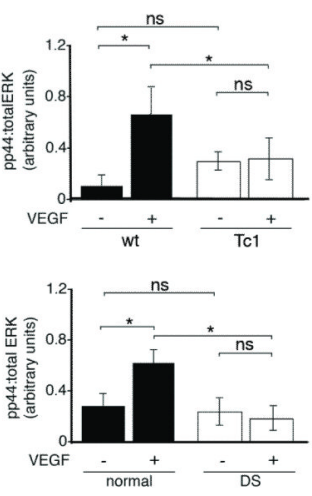

Figure 2. VEGF-mediated angiogenic responses are inhibited in Tc1 mice

a, VEGF-stimulated neovascularisation into subcutaneously implanted sponges, quantified by numbers of endomucin positive blood vessels, was decreased in Tc1 mice compared with wild-type (wt) mice. $n=20$ per group. b, VEGF-induced vessel sprouting ex vivo from Tc1 aortic rings was inhibited. Representative images of aortic ring sprouts are given. $n=6-12$ aortic rings per test. *=aortic ring, arrows = microvessel sprouts. c, Phospho-ERK1 (pp44) was increased in wild-type (wt) but not in Tc1 primary endothelial cells stimulated with VEGF. d, Phospho-ERK1 (pp44) was increased in normal human cells stimulated with VEGF but not in DS cells. $* \mathrm{P}<0.01, * * \mathrm{P}<0.05$, ns=not statistically significant. Scale bars: $100 \mu \mathrm{m}(\mathrm{a}) ; 500 \mu \mathrm{m}$ (b). All values are means \pm SEM. 

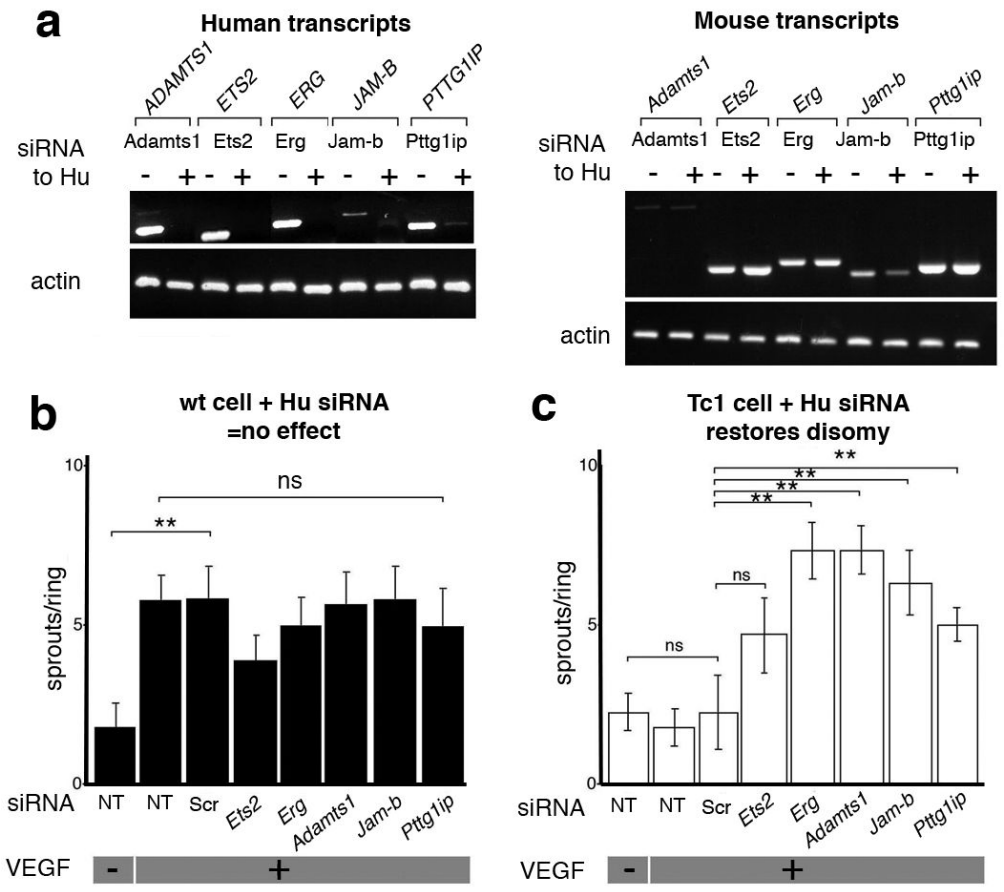

Figure 3. Reduction of copy number of candidate genes from three to two can rescue the angiogenic defect in Tc1 mice

a, siRNA depletion of the candidate human genes inhibited human transcript expression levels (left panel) but not mouse transcript expression levels (right panel). b, Nontransfected (NT) wild-type (wt) aortic rings treated with VEGF (+) enhanced microvessel sprouting compared with untreated aortic rings (-). Scrambled (Scr) siRNA or human specific siRNAs did not alter VEGF-mediated microvessel sprouting. c, Non-transfected (NT) and scrambled (Scr) siRNA transfected Tc1 aortic rings did not respond to VEGF (+). Transfection with human specific siRNAs, except ETS2, effectively restoring expression of two copies of the genes, increased VEGF-mediated vessel sprouting. $n=20-40$ aortic rings per test. Black bars: wild-type, white bars: Tc1. $* * \mathrm{P}<0.005$, ns=not statistically significant. All values are means \pm SEM. 
a

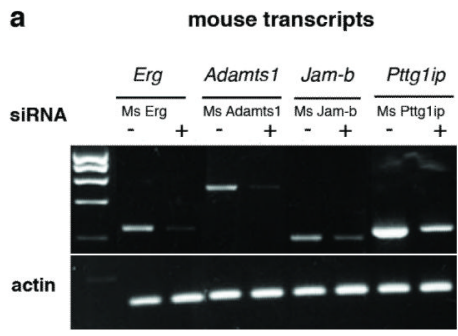

c

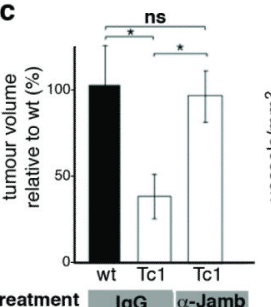

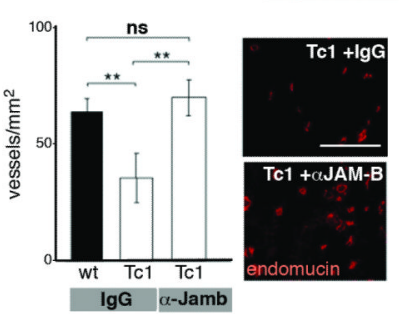

d
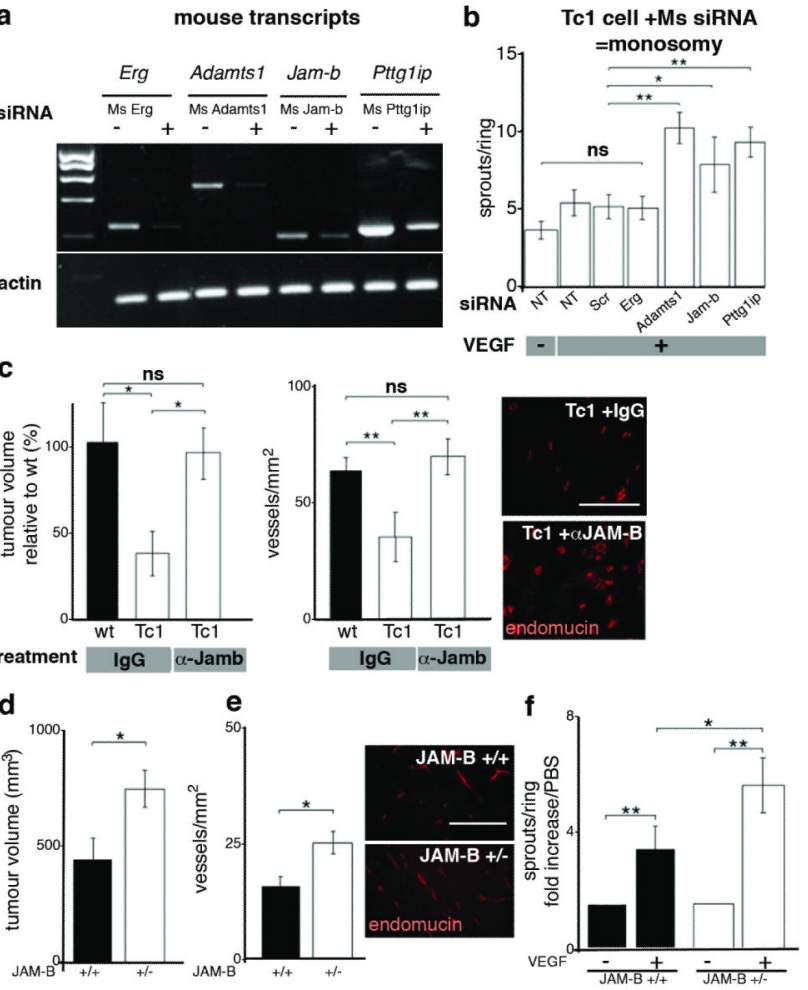

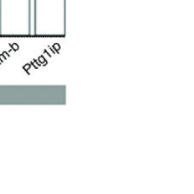

